\section{OPEN ACCESS}

Edited by:

Roland Faigle,

Johns Hopkins University,

United States

Reviewed by:

Thien J. Huynh,

Mayo Clinic Florida, United States

Jason J. Chang,

MedStar Washington Hospital Center,

United States

*Correspondence:

Si Cheng

304238@cqmu.edu.cn

Yuan Cheng

chengyuan023@aliyun.com

Specialty section:

This article was submitted to

Stroke,

a section of the journal

Frontiers in Neurology

Received: 23 July 2021 Accepted: 15 September 2021

Published: 15 October 2021

Citation:

He Q, Zhou Y, Liu C, Chen Z, Wen R,

Wu Y, Xie Z, Cheng Y and Cheng S (2021) Prediction of Hematoma

Expansion in Patients With Intracerebral Hemorrhage Using

Thromboelastography With Platelet Mapping: A Prospective Observational Study. Front. Neurol. 12:746024.

doi: 10.3389/fneur.2021.746024

\title{
Prediction of Hematoma Expansion in Patients With Intracerebral Hemorrhage Using Thromboelastography With Platelet Mapping: A Prospective Observational Study
}

\begin{abstract}
Qiuguang He ${ }^{1}$, You Zhou ${ }^{2}$, Chang Liu ${ }^{3}$, Zhongqiu Chen ${ }^{4}$, Rong Wen ${ }^{1}$, Yue Wu ${ }^{5}$, Zongyi Xie ${ }^{1}$, Yuan Cheng ${ }^{1 *}$ and Si Cheng ${ }^{6 *}$
\end{abstract}

\begin{abstract}
1 Department of Neurosurgery, The Second Affiliated Hospital of Chongqing Medical University, Chongqing, China, ${ }^{2}$ Department of Critical Care Medicine, The Second Affiliated Hospital of Chongqing Medical University, Chongqing, China, ${ }^{3}$ Department of Neurology, The Second Affiliated Hospital of Chongqing Medical University, Chongqing, China, ${ }^{4}$ Department of Information Center, The Second Affiliated Hospital of Chongqing Medical University, Chongqing, China, ${ }^{5}$ Department of Neurosurgery, The First Affiliated Hospital of Chongqing Medical University, Chongqing, China, ${ }^{6}$ Department of Orthopedics, The Second Affiliated Hospital of Chongqing Medical University, Chongqing, China
\end{abstract}

Background and Purpose: The purpose of the study was to evaluate the usefulness of thromboelastography with platelet mapping (TEG-PM) for predicting hematoma expansion (HE) and poor functional outcome in patients with intracerebral hemorrhage $(\mathrm{ICH})$.

Methods: Patients with primary $\mathrm{ICH}$ who underwent baseline computed tomography (CT) and TEG-PM within $6 \mathrm{~h}$ after symptom onset were enrolled in the observational cohort study. We performed univariate and multivariate logistic regression models to assess the association of admission platelet function with $\mathrm{HE}$ and functional outcome. In addition, a receiver operating characteristic (ROC) curve analysis investigated the accuracy of platelet function in predicting HE. A mediation analysis was undertaken to determine causal associations among platelet function, HE, and outcome.

Results: Of 142 patients, 37 (26.1\%) suffered HE. Multivariate logistic regression identified arachidonic acid (AA) and adenosine diphosphate (ADP) inhibition as significant independent predictors of HE. The area under the ROC curves was 0.727 for $A A$ inhibition and 0.721 for ADP inhibition. Optimal threshold for AA inhibition was $41.75 \% \quad(75.7 \%$ sensitivity; $67.6 \%$ specificity) and ADP inhibition was $65.8 \%$ (73.0\% sensitivity; $66.7 \%$ specificity). AA and ADP inhibition were also associated with worse 3-month outcomes after adjusting for age, admission Glasgow Coma Scale score, intraventricular hemorrhage, baseline hematoma volume, and hemoglobin. The mediation analysis showed that the effect of higher platelet inhibition with poor outcomes was mediated through HE. 
Conclusions: These findings suggest that the reduced platelet response to ADP and AA independently predict $\mathrm{HE}$ and poor outcome in patients with $\mathrm{ICH}$. Platelet function may represent a modifiable target of $\mathrm{ICH}$ treatment.

Keywords: intracerebral hemorrhage, hematoma expansion, thromboelastography, platelet function, stroke

\section{INTRODUCTION}

Spontaneous intracerebral hemorrhage (ICH) accounts for $20 \%$ of all strokes and carries the highest stroke-related morbidity and mortality $(1,2)$. Hematoma expansion (HE) usually occurs within the first few hours and is the main cause of worse functional outcome $(3,4)$. Therefore, therapeutic intervention aimed at reducing $\mathrm{HE}$ could represent a treatment paradigm in efforts to improve neurological outcome after ICH (2). However, recent $\mathrm{ICH}$ trials with hemostatic drugs, such as recombinant activated coagulation factor VII (rFVIIa) and tranexamic acid, did not reveal beneficial effects $(5,6)$. Previous studies focused on the changes of coagulation and fibrinolysis and theorized that these may contribute to HE. Actually, the role of platelet function on the occurrence of HE is insufficiently established, which may be due to the lack of effective detection methods (7).

Thromboelastography (TEG), a whole-blood viscoelastic test, offers a rapid bedside assessment of coagulability and fibrinolysis (8). Furthermore, according to the combined effect of platelet and plasma coagulation factors that contribute to hemostasis, platelet function activated by different pathways is available through TEG with platelet mapping (TEG-PM) (9). TEG-PM has previously been shown to be comparable with optical platelet aggregometry and superior to PFA-100 $(10,11)$. It is widely used in the guidance of personalized antiplatelet treatment and the assessment of perioperative period platelet function (11-13). However, there is no validated data about the level of platelet function detected by TEG-PM in patients with ICH.

The purpose of this prospective cohort study was to test the hypothesis that platelet dysfunction correlates with subsequent occurrence of $\mathrm{HE}$ and unfavorable outcome and then explore whether $\mathrm{HE}$ is the pathophysiological mechanism underlying this association. TEG-PM may be used as a clinically useful method to predict patients who will suffer $\mathrm{HE}$ and poor outcome and provide a possible direction concerning appropriate therapeutic interventions.

\section{MATERIALS AND METHODS}

\section{Patient Population}

This prospective study included spontaneous ICH patients aged 18 years or older who were admitted to our center between November 2019 and February 2021. Inclusion criteria were as follows: (1) baseline computed tomography (CT) scan was obtained within $6 \mathrm{~h}$ of symptom onset and (2) follow-up CT scan was acquired within $24 \mathrm{~h}$ of the baseline CT scan.

Exclusion criteria were as follows: (1) the presence of vascular malformation, aneurysm, traumatic, brain tumor, ischemic stroke with hemorrhagic transformation, or any other cause of secondary ICH; (2) primary intraventricular hemorrhage; (3) preceding use of antiplatelet or anticoagulant drugs; (4) receiving any hemostatic agents before TEG-PM draw; (5) surgery or other neurosurgical intervention before follow-up CT scan; (6) evidence of coagulopathy on traditional laboratory testing (14), such as activated partial thromboplastin time (APTT) $>50 \mathrm{~s}$, international normalized ratio $($ INR) $>1.5$, or platelet (PLT) count $<50 \times 10^{3} / \mu \mathrm{l}$; and (7) lost to follow-up. The Institutional Review Board of our hospital approved this study, and written informed consent was obtained from each patient or close relatives.

\section{Clinical Data and Outcome}

We prospectively collected baseline demographic and clinical data including age, sex, cerebrovascular risk factors (smoking

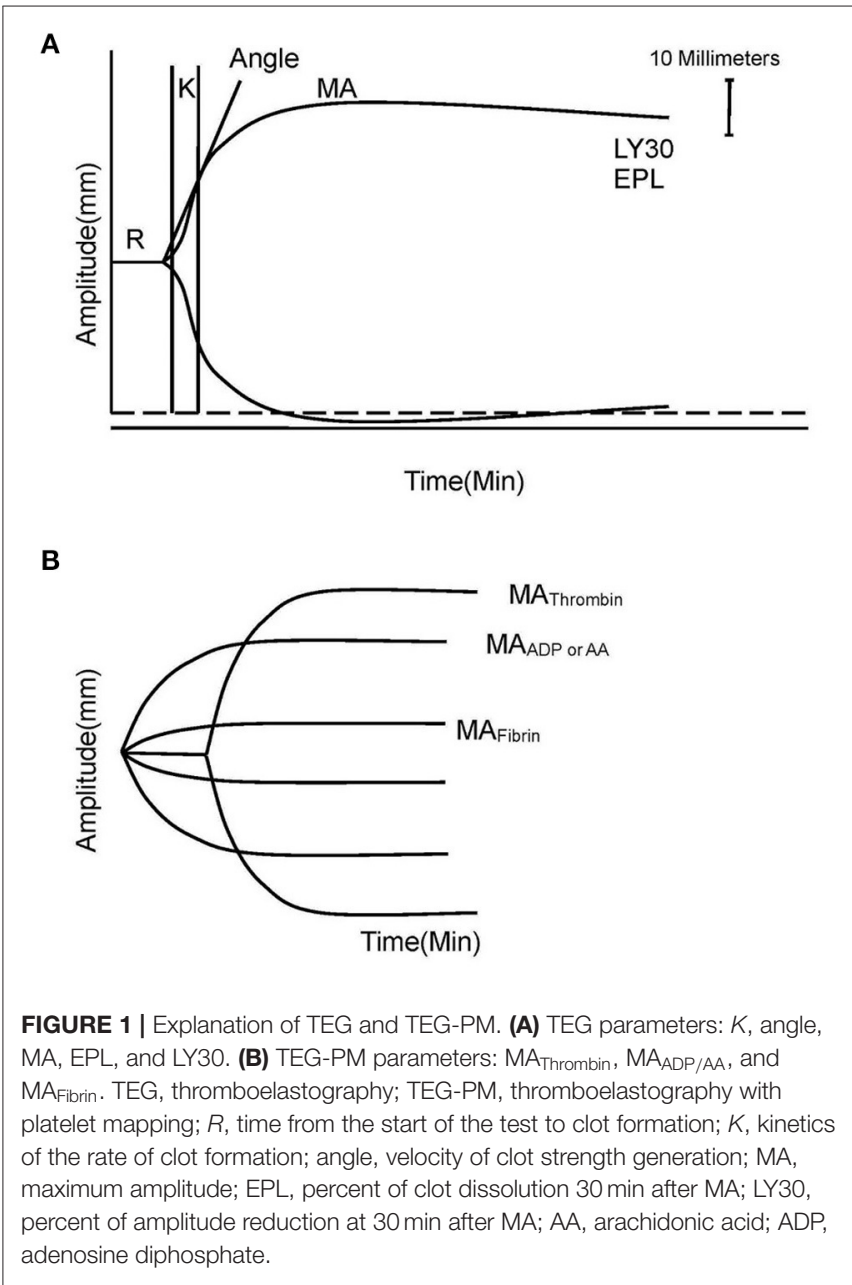


TABLE 1 | Comparison of baseline demographic, clinical characteristics, and TEG-PM parameters between patients with and without hematoma expansion.

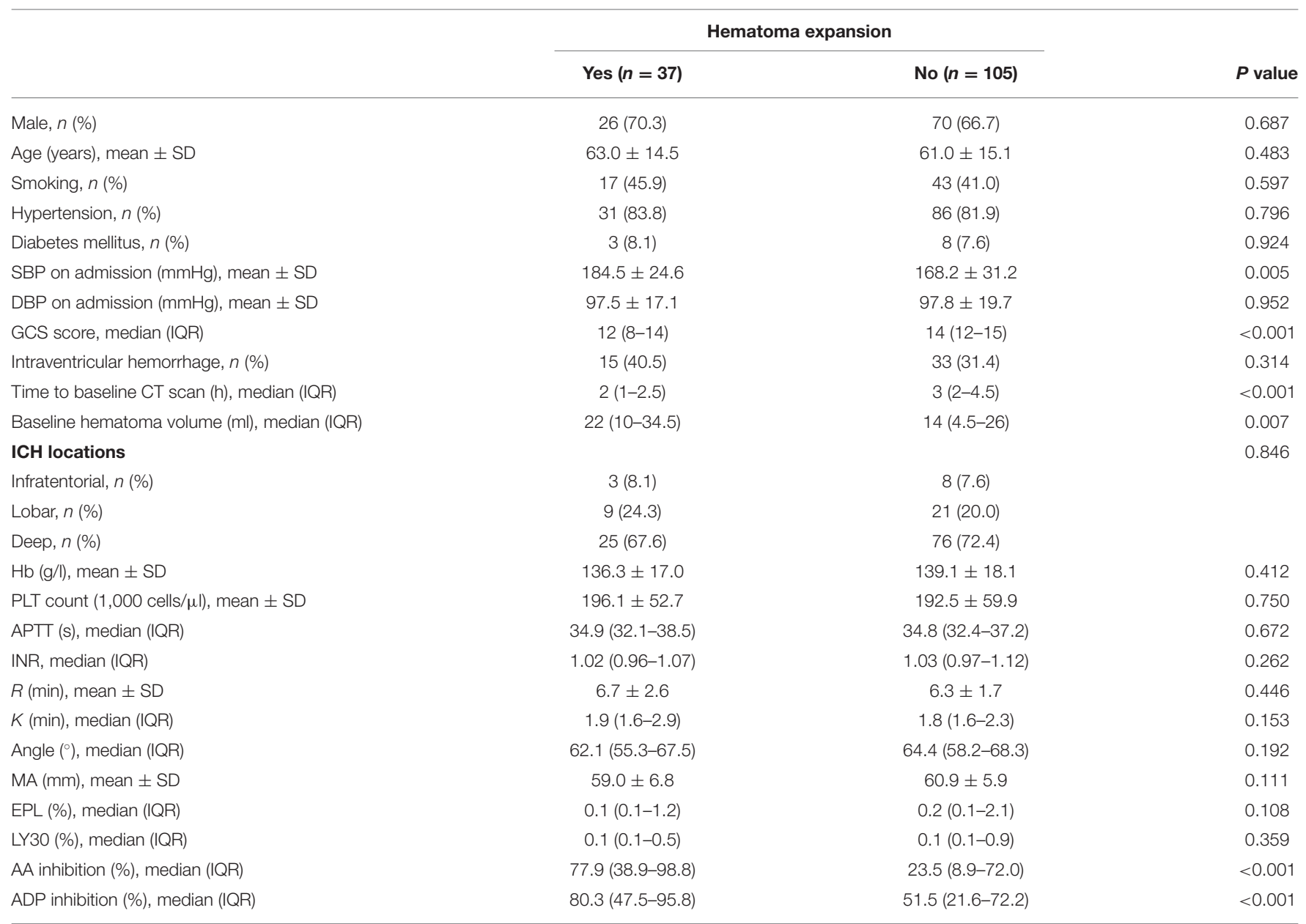

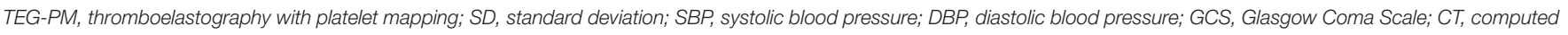

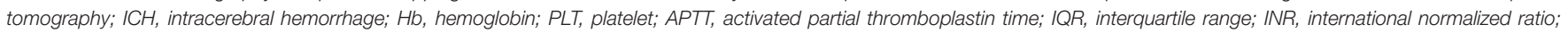

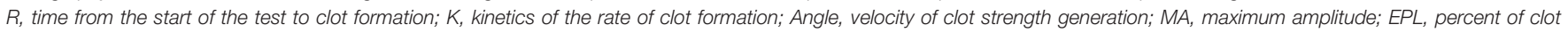
dissolution $30 \mathrm{~min}$ after MA; LY30, percent of amplitude reduction at $30 \mathrm{~min}$ after MA; $A A$, arachidonic acid; ADP, adenosine diphosphate.

status, hypertension, and diabetes mellitus), onset to first CT scan time, admission systolic blood pressure (SBP), diastolic blood pressure (DBP), and baseline Glasgow Coma Scale (GCS) score at arrival. Laboratory information included hemoglobin (Hb), PLT, APTT, and INR.

All participants received standard care according to the ICH treatment protocol of our hospital. Systolic blood pressure target was less than $140 \mathrm{mmHg}$ after admission, according to the American Heart Association/American Stroke Association guidelines (15). Functional outcome scored with the modified Rankin Scale (mRS) at 3 months was obtained by a trained research staff. Poor functional outcome was defined as a dichotomized mRS score of 3-6 according to previous studies (16-18).

\section{TEG-PM}

TEG-PM testing was performed with whole blood drawn from a single clean puncture of the median cubital vein after the diagnostic CT scan. These samples were collected in heparinized or citrated tubes and processed within $2 \mathrm{~h}$ at room temperature. The tests were done using a computerized TEG-PM analyzer (Haemoscope, Model 5000) by a trained clinical scientist. Daily quality assurance checks were carried out to ensure the validity of calibration.

The standard TEG parameters were recorded as follows: time from the start of the test to clot formation ( $R$, minutes), kinetics of the rate of clot formation ( $K$, minutes), velocity of clot strength generation (angle, degrees), maximal clot strength contributed by fibrinogen activity and platelet function (MA, millimeters), percent of amplitude reduction at $30 \mathrm{~min}$ after MA (LY30), and percent of clot dissolution 30 min after MA (EPL) (Figure 1A) (8).

The platelet inhibition shows the degree of non-response of the platelet activated by exogenous arachidonic acid (AA) and adenosine diphosphate (ADP). $\mathrm{MA}_{\text {Thrombin }}$ reflects the maximal clot strength with contribution of platelet and fibrinogen together measured by a kaolin-activated sample. $\mathrm{MA}_{\mathrm{AA}}$ and $\mathrm{MA}_{\mathrm{ADP}}$ reflect clot strength induced by stimulation of AA or ADP, 
respectively. $\mathrm{MA}_{\text {Fibrin }}$ represents individual contribution of fibrin to the clot strength measured by a reptilase and activator Factivated sample. The platelet inhibitions in response to AA and ADP were calculated with computerized software according to the equation:

$$
\begin{aligned}
100- & \left\{\left[\left(M A_{\mathrm{AA}} \text { orM } M A_{\mathrm{ADP}}\right)-M A_{\mathrm{Fibrin}}\right] /\right. \\
& \left.\left(M A_{\mathrm{Thrombin}}-M A_{\mathrm{Fibrin}}\right)\right\} \times 100(\text { Figure 1B })(19) .
\end{aligned}
$$

\section{Imaging Analysis}

According to trial protocol, a follow-up CT scan was performed within $24 \mathrm{~h}$ after the admission CT. The interval from symptom onset to admission CT was recorded. All CT scans were analyzed to determine the presence of intraventricular hemorrhage, ICH location (infratentorial, lobar, or deep), and hematoma volume. Hematoma volume measurement was performed using the 3D Slicer. Regions of interest of hematoma were identified using a semiautomatic method in each slice with a threshold range from 44 to 100 Hounsfield units. Then, we calculated hematoma volume by the accumulating volume of each slice (20-22). HE was defined as relative growth of more than $33 \%$ or an absolute growth greater than $6 \mathrm{ml}$ from initial CT according to previous studies $(3,8)$.

\section{Statistical Analysis}

Quantitative variables were summarized as mean \pm SD or as median with interquartile range (IQR) otherwise. Categorical variables were summarized as numbers with percentages. We compared baseline demographic, clinical, laboratory information, imaging characteristics, and TEG-PM parameters between patients with and without HE and then compared those between patients with good and poor outcome using $t$ test (or Mann-Whitney $U$ test for skewed distribution) for continuous variables and $\chi^{2}$ test (or Fisher's exact test) for categorical variables. To determine the independent predictors of $\mathrm{HE}$ and poor outcome, variables associated with a $P<0.10$ in univariate analyses were entered into the multivariable logistic regression models. In addition, we performed a multivariable analysis in two models because GCS and baseline hematoma volume were collinear. A mediation analysis was performed to estimate whether HE (as the mediator) was the driving factor for any relationship between platelet inhibition (independent variable) and poor outcome (dependent variable) by regressing all three variables together (23).

To obtain diagnostic threshold values of inhibition, we used receiver operator characteristic (ROC) curves considering an area under the curve (AUC) of 0.70 or higher as indicating an acceptable prediction. From the ROC curve, we determined the optimal cut-off value (with sensitivity and specificity) for discriminating the risk of HE by maximizing the Youden index (24). Statistical significance was set at a $P$ value $<0.05$. Statistical analyses were performed using SPSS 19.0 software.

\section{RESULTS}

There were $142 \mathrm{ICH}$ subjects meeting the inclusion criteria for analysis. The study population consists of $96(67.6 \%)$ men and 46
TABLE 2 | Multivariate analysis of predictors for hematoma expansion.

\begin{tabular}{lccc}
\hline & OR & $\mathbf{9 5 \%}$ Wald CI & P value \\
\hline Model 1 & & & \\
SBP on admission, mmHg & 1.016 & $0.999-1.032$ & 0.061 \\
Time to baseline CT scan, h & 0.575 & $0.417-0.793$ & 0.001 \\
Baseline hematoma volume, ml & 1.014 & $0.987-1.041$ & 0.327 \\
AA inhibition, \% & 1.020 & $1.006-1.033$ & 0.004 \\
ADP inhibition, \% & 1.026 & $1.008-1.045$ & 0.004 \\
Model 2 & & & \\
SBP on admission, mmHg & 1.012 & $0.995-1.029$ & 0.167 \\
Time to baseline CT scan, h & 0.588 & $0.424-0.815$ & 0.001 \\
GCS score & 0.861 & $0.732-1.013$ & 0.072 \\
AA inhibition, \% & 1.022 & $1.008-1.035$ & 0.001 \\
ADP inhibition, \% & 1.025 & $1.006-1.043$ & 0.008 \\
\hline
\end{tabular}

OR, odds ratio; $\mathrm{Cl}$, confidence interval; SBP, systolic blood pressure; GCS, Glasgow Coma Scale; CT, computed tomography; AA, arachidonic acid; ADP, adenosine diphosphate.

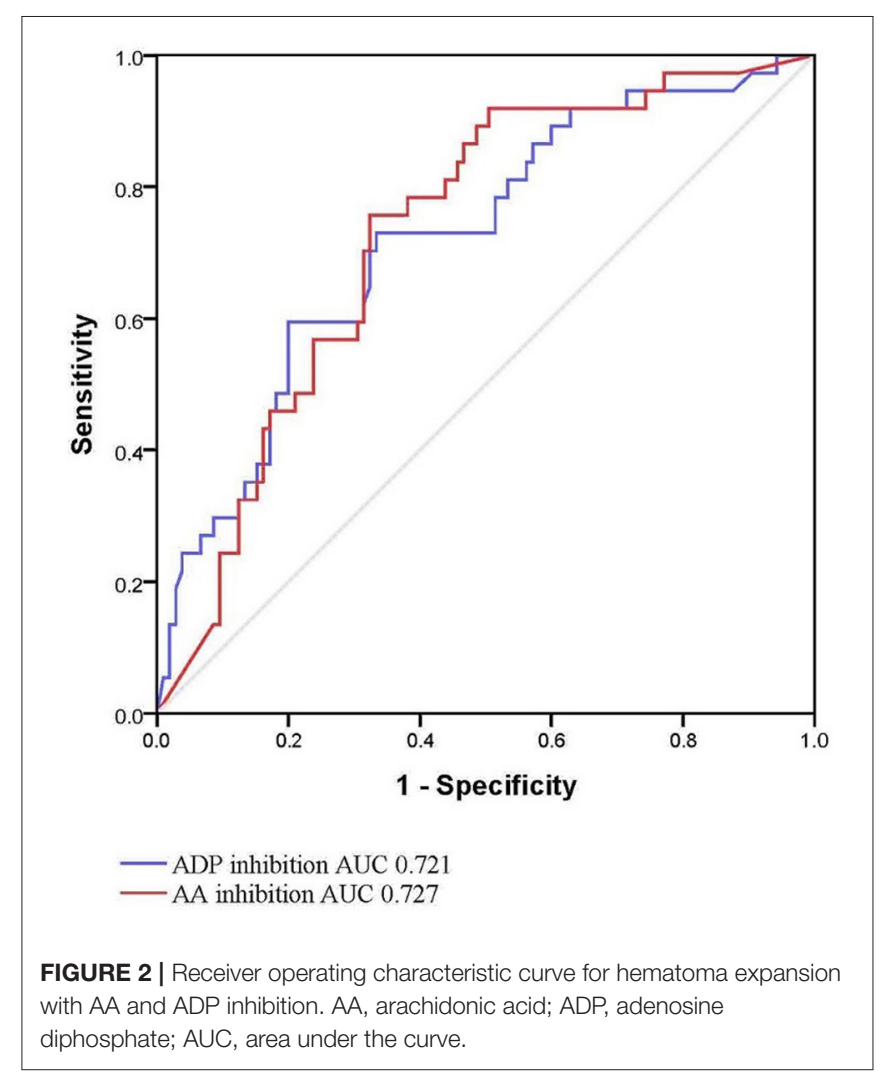

(32.4\%) women, with a mean age of 61.5 years. The median time from onset to first CT scan was $2.5 \mathrm{~h}$ (IQR, 2-4). The median baseline hematoma volume was $15 \mathrm{ml}$ (IQR, 7-27 ml), and 48 patients $(33.8 \%)$ had intraventricular hemorrhage on the first CT scan. HE occurred in $37(26.1 \%)$ cases.

Intergroup differences between ICH patients with and without HE are shown in Table 1. Subjects with HE had higher systolic blood pressure, lower admission GCS score, shorter time 
TABLE 3 | Univariate comparison between patients with good outcome (mRS 0-2) and poor outcome (mRS 3-6).

\begin{tabular}{|c|c|c|c|}
\hline & Good outcome $(n=54)$ & Poor outcome $(n=88)$ & $P$ value \\
\hline Male, $n(\%)$ & $37(68.5)$ & $59(67.0)$ & 0.856 \\
\hline Age (years), mean $\pm S D$ & $56.2 \pm 12.0$ & $64.8 \pm 15.6$ & $<0.001$ \\
\hline Smoking, $n(\%)$ & $24(44.4)$ & $36(40.9)$ & 0.679 \\
\hline Hypertension, $n$ (\%) & $43(79.6)$ & $74(84.1)$ & 0.498 \\
\hline Diabetes mellitus, $n(\%)$ & $4(7.4)$ & $7(8.0)$ & 0.906 \\
\hline GCS score, median (IQR) & $14(13-15)$ & $13(9-14)$ & $<0.001$ \\
\hline Intraventricular hemorrhage, $n$ (\%) & $12(22.2)$ & $36(40.9)$ & 0.022 \\
\hline Time to baseline CT scan (h), median (IQR) & $2(2-4)$ & $3(1.25-4)$ & 0.959 \\
\hline Baseline hematoma volume (ml), median (IQR) & $7(3-15)$ & $22.5(12-34.5)$ & $<0.001$ \\
\hline ICH locations & & & 0.216 \\
\hline $\mathrm{Hb}(\mathrm{g} / \mathrm{l})$, mean $\pm \mathrm{SD}$ & $141.5 \pm 16.6$ & $136.4 \pm 18.3$ & 0.095 \\
\hline PLT count $(1,000$ cells $/ \mu l)$, mean \pm SD & $196.7 \pm 56.0$ & $191.5 \pm 59.3$ & 0.606 \\
\hline APTT (s), median (IQR) & $34.9(32.6-37.1)$ & $34.7(32.0-37.5)$ & 0.621 \\
\hline INR, median (IQR) & $1.04(0.97-1.11)$ & $1.02(0.97-1.10)$ & 0.357 \\
\hline$R$ (min), mean $\pm \mathrm{SD}$ & $6.3 \pm 1.4$ & $6.5 \pm 2.2$ & 0.456 \\
\hline K (min), median (IQR) & $1.8(1.6-2.3)$ & $1.9(1.6-2.8)$ & 0.544 \\
\hline Angle $\left({ }^{\circ}\right)$, median (IQR) & $65.6(59.0-68.2)$ & $63.5(54.6-67.7)$ & 0.242 \\
\hline $\mathrm{MA}(\mathrm{mm})$, mean $\pm \mathrm{SD}$ & $61.4 \pm 6.6$ & $59.8 \pm 5.8$ & 0.130 \\
\hline EPL (\%), median (IQR) & $0.1(0.1-1.3)$ & $0.2(0.1-2.0)$ & 0.329 \\
\hline LY30 (\%), median (IQR) & $0.1(0.1-0.5)$ & $0.1(0.1-1.0)$ & 0.162 \\
\hline AA inhibition (\%), median (IQR) & $18.5(6.6-33.1)$ & $56.3(23.9-94.6)$ & $<0.001$ \\
\hline
\end{tabular}

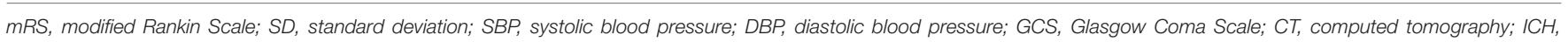

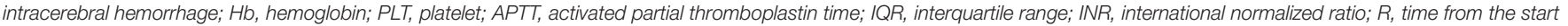

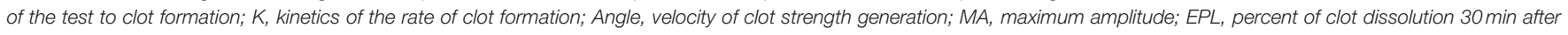
$M A ; L Y 30$, percent of amplitude reduction at $30 \mathrm{~min}$ after $M A ; A A$, arachidonic acid; $A D P$, adenosine diphosphate.

to baseline CT scan, and larger baseline hematoma volume compared to subjects without $\operatorname{HE}(P<0.05)$. There were no statistically significant differences in TEG values of $R, K$, angle, MA, EPL, and LY30 $(P>0.05)$. In multivariate logistic analyses adjusted for relevant confounders, independent predictors of $\mathrm{HE}$ were time to baseline CT scan, AA, and ADP inhibition in both models 1 and 2 (Table 2). The ability of AA and ADP inhibition to predict early $\mathrm{HE}$ is shown in Figure 2. The AUC were 0.727 (95\% CI 0.638 to 0.816 ) for AA inhibition and 0.721 (95\% CI 0.625 to 0.816 ) for ADP inhibition. We identified the optimal cutoff values of AA and ADP inhibition as $42.75 \%$ (sensitivity $75.7 \%$; specificity $67.6 \%$ ) and $65.8 \%$ (sensitivity $73.0 \%$; specificity $66.7 \%$ ) for predicting $\mathrm{HE}$, respectively.

Eighty-eight patients (62.0\%) with ICH had unfavorable outcome. The results of bivariate analysis concerning predictors of 3-month outcomes are detailed in Table 3. The age, admission GCS score, intraventricular hemorrhage, baseline hematoma volume, and AA and ADP inhibition were associated with unfavorable outcome. The multivariate logistic analysis showed that the AA and ADP inhibition remained independent predictors of unfavorable outcome in patients with $\mathrm{ICH}$ (Table 4).

When regressing platelet inhibition, $\mathrm{HE}$, and outcome together, the mediation analysis revealed that $\mathrm{HE}$ partially mediated the relationship between a high degree of AA inhibition and poor outcome. Additionally, $\mathrm{HE}$ also played a partially mediating role between $\mathrm{ADP}$ inhibition and poor outcome (Figure 3).

\section{DISCUSSION}

Our data indicate that a higher degree of AA and ADP inhibition on admission was independently associated with increased odds of HE. Furthermore, AUC suggested the acceptable performance of platelet function to predict $\mathrm{HE}$. In addition, our study demonstrated that admission platelet dysfunction was associated with poor outcome at 3 months, after adjustment for age and measures of disease severity. This relationship seemed to be mediated partly by HE. 
A previous study revealed that patients with $\mathrm{HE}$ showed longer $K$ and a trend toward longer $R$ compared with patients without HE (8). However, we were unable to identify an association of coagulation status with HE detected by TEG-PM. These differences may have been explained by the inclusion of ICH patients receiving anticoagulant or antiplatelet therapy in that study, and there were obviously more patients receiving aspirin and clopidogrel in the HE group, thus causing a slower clot formation. Additionally, the prior study did not include information on times from symptom onset to admission CT, a well-known predictor of HE. What is more, GCS score and baseline hematoma volume were not associated with $\mathrm{HE}$ in that study, which is not consistent with previously reported $\mathrm{ICH}$ cohorts $(3,25)$.

Antiplatelet medication has been demonstrated to be related to increased risk of $\mathrm{HE}$ and poor prognosis, due to the platelet dysfunction (26). Actually, high variability of platelet reactivity to $\mathrm{ADP}$ and AA in healthy volunteers has previously been reported

TABLE 4 | Multivariate analysis of predictors for poor outcome.

\begin{tabular}{lccc}
\hline & OR & 95\% Wald CI & P value \\
\hline Model 1 & & & \\
Age, years & 1.070 & $1.029-1.112$ & 0.001 \\
Intraventricular hemorrhage & 1.638 & $0.540-4.968$ & 0.383 \\
Baseline hematoma volume, ml & 1.144 & $1.078-1.214$ & $<0.001$ \\
Hb, g/l & 1.006 & $0.977-1.036$ & 0.694 \\
AA inhibition, \% & 1.019 & $1.005-1.033$ & 0.007 \\
ADP inhibition, \% & 1.027 & $1.010-1.045$ & 0.002 \\
Model 2 & & & 0.007 \\
Age, years & 1.045 & $1.012-1.080$ & 0.001 \\
GCS score & 0.679 & $0.538-0.858$ & 0.294 \\
Intraventricular hemorrhage & 1.710 & $0.627-4.664$ & 0.973 \\
Hb, g/l & 1.000 & $0.974-1.026$ & 0.002 \\
AA inhibition, \% & 1.021 & $1.008-1.034$ & 0.009 \\
ADP inhibition, \% & 1.021 & $1.005-1.036$ & \\
\hline
\end{tabular}

OR, odds ratio; Cl, confidence interval; GCS, Glasgow Coma Scale; Hb, hemoglobin; $A A$, arachidonic acid; $A D P$, adenosine diphosphate.
(11, 27), but few studies concerned about the relationship between platelet function and $\mathrm{HE}$ in $\mathrm{ICH}$ patients without antiplatelet therapy before. In our cohort, the degree of ADP and AA receptor inhibition was similar to the clinical scenario of patients taking aspirin combined with clopidogrel (11). It is unknown whether ICH itself changes platelet activity, because it is not feasible to obtain platelet activity data before and after ICH in humans. Furthermore, our multivariable analysis suggested that both AA and ADP inhibition are independent predictors of HE. However, no difference was demonstrated in platelet count, coagulation, or fibrinolysis status between the two groups.

Although our study was not designed to investigate potential pathophysiologic mechanisms of platelet function in $\mathrm{HE}$, multiple prior studies support a definitely mechanistic role for platelet function in hemostasis. When bleeding occurs, the platelet and fibrin polymer aggregate in the damaged vessel to form an immobile blood clot. Secondary mechanical shearing of peripheral vessels caused by the initial bleeding is responsible for ongoing bleeding, called $\operatorname{HE}(25,28)$. Otherwise, the accumulation of platelets activated through AA and ADP pathway subsequently enhance the hemostatic effect to prevent HE (29). This could be a possible explanation of the present study that $\mathrm{HE}$ is more likely to occur in patients with high ADP or AA inhibition.

According to the role of platelet in hemostasis, we speculate that the effect of higher platelet inhibition on outcomes in $\mathrm{ICH}$ patients is mediated through larger admission hematoma volumes and HE. This in turn results in worse functional outcomes at 3 months. In the mediation analysis, we were able to demonstrate a HE-mediated mechanism contributing to the association between platelet inhibition and functional outcome. Moreover, it is possible that mechanisms unrelated to HE may also contribute to the association.

$\mathrm{HE}$ is a clear independent predictor of increased mortality and poor functional outcomes (17). Therefore, several agents that affect the coagulation and fibrinolysis status have been investigated to prevent $\mathrm{HE}$, restricting the mass effect and secondary brain injury (30). Unfortunately, none of these clinical trials was able to reject the null hypothesis (31). In previous studies, tranexamic acid and rFVIIa reduced HE compared

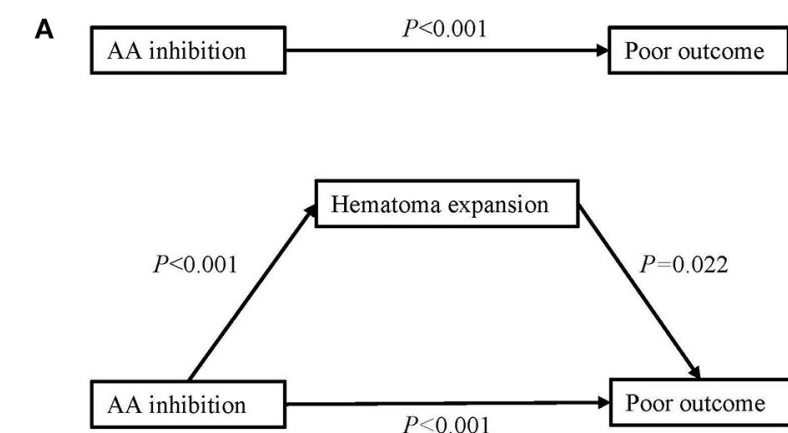

B

ADP inhibition $\quad P<0.001 \longrightarrow$ Poor outcome

FIGURE 3 | Mediation analysis of the association of platelet inhibition with poor outcome with hematoma expansion as a mediator. (A) Mediation analysis of AA inhibition in poor outcome by hematoma expansion. (B) Mediation analysis of ADP inhibition in poor outcome by hematoma expansion. AA, arachidonic acid; ADP, adenosine diphosphate. 
with placebo, but did not improve survival or functional outcomes $(6,32)$. Our data may suggest that improvement of platelet function can be considered in preventing the early occurrence of $\mathrm{HE}$ to improve outcome. However, a previous study showed that platelet transfusion did not reduce $\mathrm{HE}$ for people taking antiplatelet therapy before ICH. On the contrary, it was associated with increased mortality and dependence in 3 months (18). One possible explanation is that HE is more closely associated with platelet function than platelet count itself, as demonstrated in our multivariable analysis and a previous cohort study (33). Another possible explanation is that the harmful effects were partly caused by transfusing $\mathrm{ABO}$-incompatible platelets (34). Two prior studies have investigated the effect of desmopressin on improving platelet function in ICH patients and found that intravenous desmopressin was well tolerated and obviously improved platelet activity $(35,36)$. According to the results of our study, it is reasonable to believe that desmopressin may be a potential pharmacological treatment in preventing $\mathrm{HE}$ to improve outcome.

Thus far, clinical trials aimed at restricting expansion have not led to improved neurological outcome $(6,18)$, perhaps because such therapies need to be targeted to patients at highest risk for expansion to show any benefit. So, effective biomarkers are needed to select patients and guide interventions to restrict HE. The computed tomographic angiography (CTA) spot sign, a novel radiographic marker, has proven to be a promising predictor of $\mathrm{HE}$ with a sensitivity of $51 \%$ and a specificity of $85 \%$ (3). In our study, the sensitivity and specificity of platelet inhibition for predicting expansion were 73.0 and $66.7 \%$ for ADP inhibition and 75.7 and $67.6 \%$ for AA inhibition, respectively. Compared with the CTA spot sign, platelet inhibition has a lower specificity, but it is more sensitive for predicting HE. Moreover, CTA spot sign was just an imaging sign that cannot be altered, while platelet function can be directly improved. Additionally, recent clinical trials did not show reduced $\mathrm{HE}$ or improved clinical outcomes in spot sign-positive ICH patients through the use of rFVIIa or tranexamic acid $(37,38)$. Thus, it may be infeasible to use CTA spot sign as a predictor to target hemostatic therapy in acute ICH patients. Our data may suggest that TEG$\mathrm{PM}$ is useful to identify individuals in high risk for HE, and they should receive aggressive treatments such as desmopressin and hemostatic drugs.

Inherent limitations of our analysis should be clarified. Our study was a single-center observational cohort with limited

\section{REFERENCES}

1. Qureshi AI, Mendelow AD, Hanley DF. Intracerebral haemorrhage. Lancet. (2009) 373:1632-44. doi: 10.1016/S0140-6736(09)60371-8

2. Keep RF, Hua Y, Xi G. Intracerebral haemorrhage: mechanisms of injury and therapeutic targets. Lancet Neurol. (2012) 11:72031. doi: 10.1016/S1474-4422(12)70104-7

3. Demchuk AM, Dowlatshahi D, Rodriguez-Luna D, Molina CA, Blas YS, Dzialowski I, et al. Prediction of haematoma growth and outcome in patients with intracerebral haemorrhage using the CT-angiography spot sign (PREDICT): a prospective observational study. Lancet Neurol. (2012) 11:307-14. doi: 10.1016/S1474-4422(12)70038-8

4. Davis SM, Broderick J, Hennerici M, Brun NC, Diringer MN, Mayer $\mathrm{SA}$, et al. Hematoma growth is a determinant of mortality and sample size and will need to be replicated. In addition, TEG-PM was tested only once on admission. It is unclear whether there are dynamical changes in platelet function, which might explain the reason why $\mathrm{HE}$ often occurs in early stage. Finally, we did not perform CTA spot sign testing and the correlation of platelet function with the CTA spot sign was not analyzed.

Further investigation is warranted to confirm our findings of more $\mathrm{HE}$ after $\mathrm{ICH}$ in patients with lower platelet function, resulting in worse clinical outcomes. If confirmed, these findings may suggest the importance of accounting for and correcting platelet dysfunction in future ICH treatment strategies for $\mathrm{HE}$ in efforts to improve outcome.

\section{DATA AVAILABILITY STATEMENT}

The raw data supporting the conclusions of this article will be made available by the authors, without undue reservation.

\section{ETHICS STATEMENT}

The studies involving human participants were reviewed and approved by Medical Ethics Committee of the Second Affiliated Hospital of Chongqing Medical University. The patients/participants provided their written informed consent to participate in this study.

\section{AUTHOR CONTRIBUTIONS}

$\mathrm{QH}, \mathrm{YC}$, and SC conceived and designed the study. QH, YZ, $\mathrm{CL}, \mathrm{RW}$, and YW acquired the data, which ZC analyzed. $\mathrm{QH}, \mathrm{YZ}$, and ZX aided in data interpretation and wrote the manuscript. All authors contributed to the article and approved the submitted version.

\section{FUNDING}

This work was supported by Chongqing Medical Scientific Research Project (No. 2022WSJK036).

\section{ACKNOWLEDGMENTS}

We thank all the participants for their important contributions to our study. This manuscript has not been published elsewhere in whole or in part.

poor outcome after intracerebral hemorrhage. Neurology.

(2006) 66:1175-81. doi: 10.1212/01.wnl.0000208408.98482.99

5. Al-Shahi Salman R, Law ZK, Bath PM, Steiner T, Sprigg N. Haemostatic therapies for acute spontaneous intracerebral haemorrhage. Cochrane Datab Systemat Rev. (2018) 4:CD005951. doi: 10.1002/14651858.CD005951.pub4

6. Sprigg N, Flaherty K, Appleton JP, Al-Shahi Salman R, Bereczki $\mathrm{D}$, Beridze $\mathrm{M}$, et al. Tranexamic acid for hyperacute primary IntraCerebral Haemorrhage (TICH-2): an international randomised, placebo-controlled, phase 3 superiority trial. Lancet. (2018) 391:2107-15. doi: 10.1016/S0140-6736(18)31033-X

7. Burchell SR, Tang J, Zhang JH. Hematoma expansion following intracerebral hemorrhage: mechanisms targeting the coagulation cascade and platelet activation. Curr Drug Targets. (2017) 18:1329-44. doi: 10.2174/1389450118666170329152305 
8. Kawano-Castillo J, Ward E, Elliott A, Wetzel J, Hassler A, McDonald M, et al. Thrombelastography detects possible coagulation disturbance in patients with intracerebral hemorrhage with hematoma enlargement. Stroke. (2014) 45:683-8. doi: 10.1161/STROKEAHA.113.003826

9. He Q, Zhou Y, Liu C, Zhang X, Huang N, Wang F, et al. Thromboelastography with platelet mapping detects platelet dysfunction in patients with aneurysmal subarachnoid hemorrhage with rebleeding. Neuropsychiatr Dis Treat. (2019) 15:3443-51. doi: 10.2147/NDT.S229284

10. Agarwal S, Coakley M, Reddy K, Riddell A, Mallett S. Quantifying the effect of antiplatelet therapy: a comparison of the platelet function analyzer (PFA-100) and modified thromboelastography (mTEG) with light transmission platelet aggregometry. Anesthesiology. (2006) 105:67683. doi: 10.1097/00000542-200610000-00011

11. Collyer TC, Gray DJ, Sandhu R, Berridge J, Lyons G. Assessment of platelet inhibition secondary to clopidogrel and aspirin therapy in preoperative acute surgical patients measured by thrombelastography platelet mapping. $\mathrm{Br} \mathrm{J}$ Anaesth. (2009) 102:492-8. doi: 10.1093/bja/aep039

12. Kasivisvanathan R, Abbassi-Ghadi N, Kumar S, Mackenzie H, Thompson $\mathrm{K}$, James $\mathrm{K}$, et al. Risk of bleeding and adverse outcomes predicted by thromboelastography platelet mapping in patients taking clopidogrel within 7 days of non-cardiac surgery. Br J Surg. (2014) 101:138390. doi: 10.1002/bjs.9592

13. Zhao X, Li Q, Tu C, Zeng Y, Ye Y. High glycated albumin is an independent predictor of low response to clopidogrel in ACS patients: a cross-sectional study. Cardiovasc Diabetol. (2020) 19:171. doi: 10.1186/s12933-020-01146-w

14. Roh D, Torres GL, Cai C, Zammit C, Reynolds AS, Mitchell A, et al. Coagulation differences detectable in deep and lobar primary intracerebral hemorrhage using thromboelastography. Neurosurgery. (2020) 87:91824. doi: 10.1093/neuros/nyaa056

15. Hemphill JC 3rd, Greenberg SM, Anderson CS, Becker K, Bendok BR, Cushman $\mathrm{M}$, et al. Guidelines for the management of spontaneous intracerebral hemorrhage: a guideline for healthcare professionals from the American Heart Association/American Stroke Association. Stroke. (2015) 46:2032-60. doi: 10.1161/STR.0000000000000069

16. Anderson CS, Heeley E, Huang Y, Wang J, Stapf C, Delcourt C, et al. Rapid blood-pressure lowering in patients with acute intracerebral hemorrhage. $N$ Engl J Med. (2013) 368:2355-65. doi: 10.1056/NEJMoa1214609

17. Selim M, Foster LD, Moy CS Xi G, Hill MD, Morgenstern LB, et al. Deferoxamine mesylate in patients with intracerebral haemorrhage (i-DEF): a multicentre, randomised, placebo-controlled, double-blind phase 2 trial. Lancet Neurol. (2019) 18:428-38. doi: 10.1016/S1474-4422(19)30069-9

18. Baharoglu MI, Cordonnier C, Al-Shahi Salman R, de Gans K, Koopman MM, Brand A, et al. Platelet transfusion versus standard care after acute stroke due to spontaneous cerebral haemorrhage associated with antiplatelet therapy (PATCH): a randomised, open-label, phase 3 trial. Lancet. (2016) 387:2605-13. doi: 10.1016/S0140-6736(16)30392-0

19. Gurbel PA, Bliden KP, Tantry US, Monroe AL, Muresan AA, Brunner NE, et al. First report of the point-of-care TEG: a technical validation study of the TEG6S system. Platelets. (2016) 27:642-9. doi: 10.3109/09537104.2016.1153617

20. Xu X, Chen X, Li F, Zheng X, Wang Q, Sun G, et al. Effectiveness of endoscopic surgery for supratentorial hypertensive intracerebral hemorrhage: a comparison with craniotomy. J Neurosurg. (2018) 128:553-9. doi: 10.3171/2016.10.JNS161589

21. Chen M, Li Z, Ding J, Lu X, Cheng Y, Lin J. Comparison of common methods for precision volume measurement of hematoma. Comput Math Methods Med. (2020) 2020:6930836. doi: 10.1155/2020/6930836

22. Xu X, Chen X, Zhang J, Zheng Y, Sun G, Yu X, et al. Comparison of the Tada formula with software slicer: precise and low-cost method for volume assessment of intracerebral hematoma. Stroke. (2014) 45:34335. doi: 10.1161/STROKEAHA.114.007095

23. Preacher KJ, Hayes AF, SPSS. and SAS procedures for estimating indirect effects in simple mediation models. Behav Res Methods Instrum Comput. (2004) 36:717-31. doi: 10.3758/BF03206553

24. Hanley JA, McNeil BJ. The meaning and use of the area under a receiver operating characteristic (ROC) curve. Radiology. (1982) 143:2936. doi: 10.1148/radiology.143.1.7063747

25. Boulouis G, Morotti A, Brouwers HB, Charidimou A, Jessel MJ, Auriel E, et al. Association between hypodensities detected by computed tomography and hematoma expansion in patients with intracerebral hemorrhage. JAMA Neurol. (2016) 73:961-8. doi: 10.1001/jamaneurol.2016.1218

26. Thompson BB, Bejot Y, Caso V, Castillo J, Christensen H, Flaherty ML, et al. Prior antiplatelet therapy and outcome following intracerebral hemorrhage: a systematic review. Neurology. (2010) 75:1333-42. doi: 10.1212/WNL.0b013e3181f735e5

27. Bochsen L, Wiinberg B, Kjelgaard-Hansen M, Steinbruchel DA, Johansson PI. Evaluation of the TEG platelet mapping assay in blood donors. Thromb J. (2007) 5:3. doi: 10.1186/1477-9560-5-3

28. Schlunk F, Greenberg SM. The pathophysiology of intracerebral hemorrhage formation and expansion. Transl Stroke Res. (2015) 6:257-63. doi: 10.1007/s12975-015-0410-1

29. Siddiqui TI, Kumar KSA, Dikshit DK. Platelets and atherothrombosis: causes, targets and treatments for thrombosis. Curr Med Chem. (2013) 20:277997. doi: 10.2174/0929867311320220004

30. Rincon F, Mayer SA. Intracerebral hemorrhage: clinical overview and pathophysiologic concepts. Transl Stroke Res. (2012) 3:10-24. doi: 10.1007/s12975-012-0175-8

31. Al-Kawaz MN, Hanley DF, Ziai W. Advances in therapeutic approaches for spontaneous intracerebral hemorrhage. Neurotherapeutics. (2020) 17:175767. doi: 10.1007/s13311-020-00902-w

32. Mayer SA, Brun NC, Begtrup K, Broderick J, Davis S, Diringer $\mathrm{MN}$, et al. Efficacy and safety of recombinant activated factor VII for acute intracerebral hemorrhage. $N$ Engl J Med. (2008) 358:2127-37. doi: 10.1056/NEJMoa0707534

33. Mrochen A, Sprugel MI, Gerner ST, Sembill JA, Lang S, Lucking $\mathrm{H}$, et al. Thrombocytopenia and clinical outcomes in intracerebral hemorrhage: a retrospective multicenter cohort study. Stroke. (2021) 52:6119. doi: 10.1161/STROKEAHA.120.031478

34. Magid-Bernstein J, Beaman CB, Carvalho-Poyraz F, Boehme A, Hod EA, Francis RO, et al. Impacts of ABO-incompatible platelet transfusions on platelet recovery and outcomes after intracerebral hemorrhage. Blood. (2021) 137:2699-703. doi: 10.1182/blood.2020008381

35. Naidech AM, Maas MB, Levasseur-Franklin KE, Liotta EM, Guth JC, Berman M, et al. Desmopressin improves platelet activity in acute intracerebral hemorrhage. Stroke. (2014) 45:2451-3. doi: 10.1161/STROKEAHA.114.006061

36. Kapapa T, Rohrer S, Struve S, Petscher M, Konig R, Wirtz CR, et al. Desmopressin acetate in intracranial haemorrhage. Neurol Res Int. (2014) 2014:298767. doi: 10.1155/2014/298767

37. Gladstone DJ, Aviv RI, Demchuk AM, Hill MD, Thorpe KE, Khoury JC, et al. Effect of recombinant activated coagulation factor VII on hemorrhage expansion among patients with spot sign-positive acute intracerebral hemorrhage: the SPOTLIGHT and STOP-IT randomized clinical trials. JAMA Neurol. (2019) 76:1493-501. doi: 10.1001/jamaneurol.2019.2636

38. Meretoja A, Yassi N, Wu TY, Churilov L, Sibolt G, Jeng JS, et al. Tranexamic acid in patients with intracerebral haemorrhage (STOP-AUST): a multicentre, randomised, placebo-controlled, phase 2 trial. The Lancet Neurology. (2020) 19:980-7. doi: 10.1016/S1474-4422(20)30369-0

Conflict of Interest: The authors declare that the research was conducted in the absence of any commercial or financial relationships that could be construed as a potential conflict of interest.

Publisher's Note: All claims expressed in this article are solely those of the authors and do not necessarily represent those of their affiliated organizations, or those of the publisher, the editors and the reviewers. Any product that may be evaluated in this article, or claim that may be made by its manufacturer, is not guaranteed or endorsed by the publisher.

Copyright (C) $2021 \mathrm{He}$, Zhou, Liu, Chen, Wen, Wu, Xie, Cheng and Cheng. This is an open-access article distributed under the terms of the Creative Commons Attribution License (CC BY). The use, distribution or reproduction in other forums is permitted, provided the original author(s) and the copyright owner(s) are credited and that the original publication in this journal is cited, in accordance with accepted academic practice. No use, distribution or reproduction is permitted which does not comply with these terms. 